\title{
An Astigmatic Detection System for Polymeric Cantilever-Based Sensors
}

\author{
En-Te Hwu, ${ }^{1}$ Hsien-Shun Liao, ${ }^{1,2}$ Filippo G. Bosco, ${ }^{3}$ Ching-Hsiu Chen, ${ }^{1}$ \\ Stephan Sylvest Keller, ${ }^{3}$ Anja Boisen, ${ }^{3}$ and Kuang-Yuh Huang ${ }^{2}$ \\ ${ }^{1}$ Institute of Physics, Academia Sinica, Nankang, Taipei 11529, Taiwan \\ ${ }^{2}$ Department of Mechanical Engineering, National Taiwan University, Taipei 10617, Taiwan \\ ${ }^{3}$ Department of Micro- and Nanotechnology, Technical University of Denmark, 2800 Lyngby, Denmark
}

Correspondence should be addressed to En-Te Hwu, whoand@phys.sinica.edu.tw

Received 15 July 2011; Accepted 20 August 2011

Academic Editor: Martin Hegner

Copyright () 2012 En-Te Hwu et al. This is an open access article distributed under the Creative Commons Attribution License, which permits unrestricted use, distribution, and reproduction in any medium, provided the original work is properly cited.

\begin{abstract}
We demonstrate the use of an astigmatic detection system (ADS) for resonance frequency identification of polymer microcantilever sensors. The ADS technology is based on a DVD optical head combined with an optical microscope (OM). The optical head has a signal bandwidth of $80 \mathrm{MHz}$, allowing thermal fluctuation measurements on cantilever beams with a subnanometer resolution. Furthermore, an external excitation can intensify the resonance amplitude, enhancing the signal- to-noise ratio. The full width at half maximum (FWHM) of the laser spot is $568 \mathrm{~nm}$, which facilitates read-out on potentially submicrometer-sized cantilevers. The resonant frequency of SU-8 microcantilevers is measured by both thermal fluctuation and excited vibration measurement modes of the ADS.
\end{abstract}

\section{Introduction}

Cantilever-based sensors have emerged as a promising labelfree detection technique, which have been used for highprecision mass detection and biomolecular recognition. By surface functionalization, the cantilever can be modified specific to certain compounds detection. Molecules adsorbed to one side of the cantilever will deflect the cantilever due to changes in surface stress [1-3]. Alternatively, minute mass changes can be detected by monitoring the resonant frequency change of the cantilever for high-precision mass detections [4]. By monitoring surface stress changes, for example, DNA hybridization [5] and antibiotic-peptide binding [6] have been detected.

For detecting the vibrational amplitude and/or the deflection of a microcantilever, diverse methods such as optical [7], capacitive [8], piezoresistive and piezoelectric [911] have been applied. The optical lever technique, typically used in atomic force microscopy (AFM), is the most popular method for detecting deflections of micromechanical structures. A laser beam is focused on a microcantilever which reflects the beam onto a position-sensitive detector (PSD).
The distance between the microcantilever and the PSD magnifies the angular detection sensitivity that subnanometer cantilever deflections can be resolved [12, 13]. The optical lever technique needs a tedious and time-consuming adjustment process before measuring each cantilever own bending angle. Therefore, the optical lever method may not be an optimum solution for new technological tasks such as fast or simultaneous detection on large microcantilever arrays. Moreover, the laser spot size in most commercially available optical lever systems is several micrometers, which is difficult to measure submicrometer-sized structures.

Previously, an astigmatic detection system (ADS) is applied in an AFM system [14] for monitoring the AFM probes. The key component of the ADS is an optical head inside a DVD ROM drive. We have demonstrated that the atomic-scale thermal noise of an AFM probe can be measured by the ADS. In this work, we combine the ADS with an optical microscope (OM) for measuring microcantilever-based sensors made of polymer SU-8 [15].

The detection scheme of the ADS is shown in Figure 1(a). Through a collimator and an objective lens, a laser beam 


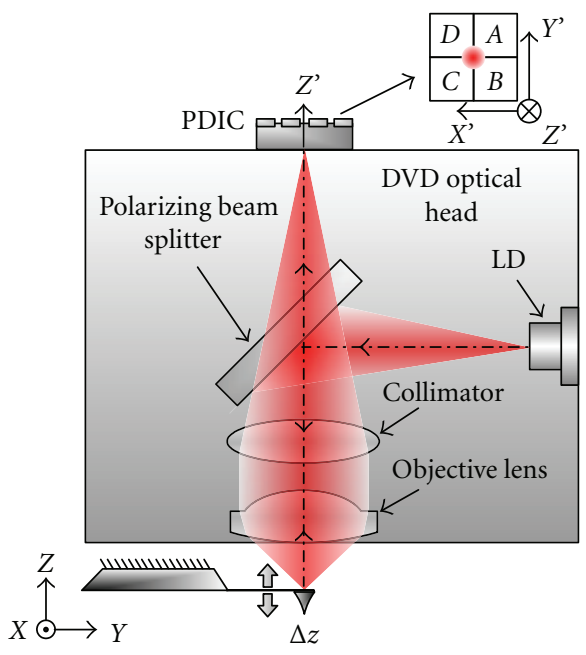

(a)

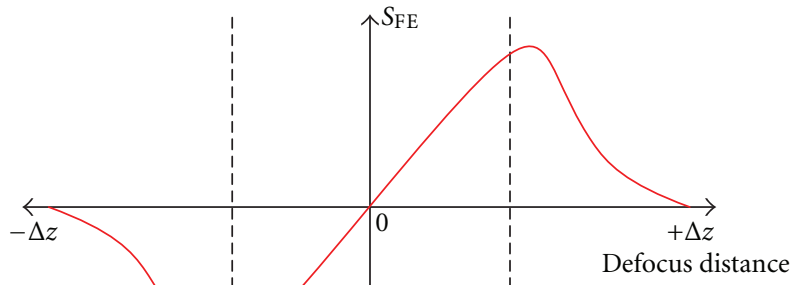

$\underbrace{}_{\downarrow}$

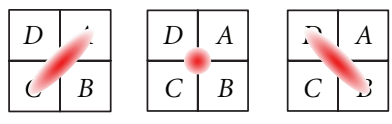

$S_{\mathrm{FE}}=\left(S_{A}+S_{C}\right)-\left(S_{B}+S_{D}\right)$

(b)

FIGURE 1: Detection scheme of the astigmatic detection system, (a) optical path configuration, (b) laser spot on PDIC, and the focus error signal; the linear region is about $6 \mu \mathrm{m}$.

generated by a laser diode (LD) is focused onto a cantilever. The cantilever reflects the laser beam back through a beam splitter, which perpendicularly reflects the laser beam onto the photodetector integrated chip (PDIC). The optoelectronic energy transformation is carried out by four independent photosensitive quadrants $(A, B, C, D)$, which generate the signals of $S_{A}, S_{B}, S_{C}$, and $S_{D}$, respectively. When the ADS is focused on the cantilever, the laser spot on PDIC is circular shaped. The defocus distance $\Delta z$ of the object induces a shape change of the laser spot, as shown in Figure 1(b). A corresponding focus error signal $S_{\mathrm{FE}}$ is defined as $S_{\mathrm{FE}}=\left(S_{A}+S_{C}\right)-\left(S_{B}+S_{D}\right)$. For monitoring and accurate aligning the laser spot on the object, an OM with CMOS sensor, is combined to the optical path of the ADS.

Without angular adjustment, the ADS can still measure resonance frequencies of cantilevers with bending angle from -8- to 8-degree deviation. Compared with the optical lever technique, the large angular tolerance of the ADS makes high-speed measurement possible [16]. One of the main challenges is to be able to measure the resonant peak of the polymer cantilevers without reflective coatings. This would represent a very useful tool for employing the technology to out-of-the-lab applications, drastically reducing the size and cost of the readout setup. Furthermore, the SU-8 microcantilevers without coating can substantially simplify the manufacturing process and reduce the cost of the microcantilever-based biosensor. In this paper, we measure the resonant frequency of the microcantilevers in four conditions: with and without reflective coating and with and without external excitation.

\section{SU-8 Microcantilevers Dynamic Analysis}

Our developed microcantilevers are made of polymer SU8 , which possesses the advantages of simple processing, low Young's modulus for high force resolution and low sensitivity

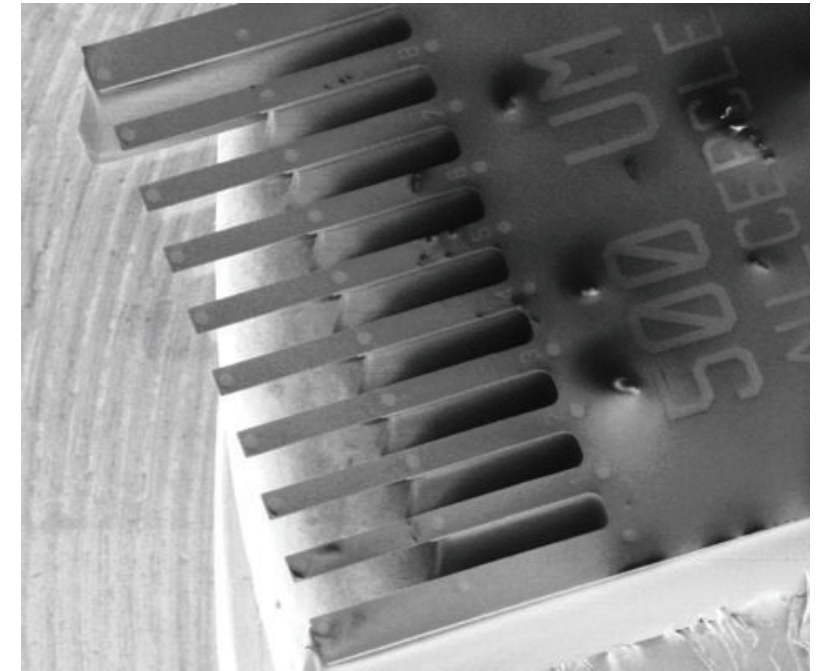

FIGURE 2: SEM image of eight SU-8 microcantilevers with gold pads.

to the environmental temperature [17]. Figure 2 shows an SEM image of eight SU-8 microcantilevers on one chip. Each microcantilever is $495 \mu \mathrm{m}$ long, $100 \mu \mathrm{m}$ wide, and $5.3 \mu \mathrm{m}$ thick. On the free end of each microcantilever is a circular coated gold pad with a diameter of $50 \mu \mathrm{m}$ and a thickness of $20 \mathrm{~nm}$. The gold pads provide well-reflective surfaces for the optical detection.

In order to validate the performance of the ADS, analytical models and finite element methods (FEM-) are used to calculate the resonant frequencies of the SU-8 microcantilevers. Their mechanical properties are mainly influenced by shape, materials, and geometrical sizes. The spring constant and the fundamental resonant frequency of 
TABLE 1: Mechanical properties and dimensions of an SU-8 microcantilever.

\begin{tabular}{lcc}
\hline$E$ & Young's modulus & $4.2 \pm 0.5 \mathrm{G} \mathrm{N} / \mathrm{m}^{2}$ \\
\hline$\rho$ & Density & $1200 \mathrm{~kg} / \mathrm{m}^{3}$ \\
\hline$L$ & Length & $495 \mu \mathrm{m}$ \\
\hline$W$ & Width & $100 \mu \mathrm{m}$ \\
\hline$T$ & Thickness & $5.3 \pm 0.1 \mu \mathrm{m}$ \\
\hline
\end{tabular}

TABLE 2: Simulated resonant frequencies by using FEMLAB.

\begin{tabular}{ll}
\hline First bending mode $f_{\text {1st }}$ & $6.59 \pm 0.5 \mathrm{kHz}$ \\
\hline Second bending mode $f_{\text {2nd }}$ & $41.3 \pm 3.3 \mathrm{kHz}$ \\
\hline Torsional mode $f_{T}$ & $66.2 \pm 5.2 \mathrm{kHz}$ \\
\hline
\end{tabular}

a homogeneous rectangular microcantilever in vacuum can be approximated by (1) and (2), respectively [18]

$$
\begin{gathered}
k=\frac{E w t^{3}}{4 L^{3}}, \\
f_{1 \mathrm{st}}=0.162 \sqrt{\frac{E}{\rho}} \frac{t}{L^{2}} .
\end{gathered}
$$

Table 1 lists the mechanical properties and geometrical sizes of the SU-8 microcantilevers [19]. Based on these values, the calculated spring constant $k$ and resonant frequency $f_{1 s t}$ of the microcantilever are $0.128 \pm 0.02 \mathrm{~N} / \mathrm{m}$ and $6.55 \pm 0.4 \mathrm{kHz}$, respectively.

By using finite element analysis software (FEMLAB), simulated resonant frequencies are listed in Table 2. The first bending mode frequency of the microcantilever is $6.43 \mathrm{kHz}$, which well-matches the theoretical value $6.55 \pm 0.4 \mathrm{kHz}$ calculated by (2).

The resonant frequency of the microcantilever is shifted by its mass change due to absorption of specific target molecules. For realizing a high mass sensitivity, the binding region for the target molecule can be confined to the free end of the microcantilever. The bound mass variation $\Delta m$ can be associated with $f_{1 s t}$ and the frequency variation $\Delta f$, as shown in

$$
\Delta m=\frac{k}{4 \pi^{2}}\left(\frac{1}{\left(f_{\mathrm{lst}}+\Delta f\right)^{2}}-\frac{1}{f_{\mathrm{lst}}^{2}}\right) .
$$

When $\Delta f$ and the $\Delta m$ are close to zero, (3) can be simplified as (4), which describes the sensitivity of the mass variation $\Delta m$ to the frequency variation $\Delta f$

$$
\frac{\Delta m}{\Delta f}=-\frac{k}{2 \pi^{2} f_{1 \mathrm{st}}{ }^{3}} .
$$

From the calculated spring constant $k=0.128 \pm 0.02 \mathrm{~N} / \mathrm{m}$ and resonant frequency $f_{1 \text { st }} 6.55 \pm 0.4 \mathrm{kHz}$, we can derive a sensitivity $\Delta m / \Delta f$ of $-23.2 \mathrm{pg} / \mathrm{Hz}$. Higher resonant frequency of the microcantilever can significantly enhance the measurement resolution of the mass. For a constant sampling rate, increasing the number of sampling data can also improve the measurement resolution of frequency, but it will slow down the measurement speed.

\section{Experimental Setup}

Figure 3 illustrates a block diagram of the ADS combined with an OM. Through an optomechanical adaptor, the ADS can be easily attached to the objective of the OM (Nikon Eclipse E100). The CMOS camera is employed to capture a magnified image of the cantilever and display the image on the PC monitor for controlling the alignment process. A light source from the bottom can illuminate the microcantilevers for enhancing the contrast of the optical image.

The ADS is based on a slim type DVD optical head which has a size of $50 \times 35 \times 6 \mathrm{~mm}$. The laser diode of the ADS generates a laser beam with a wavelength of $655 \mathrm{~nm}$. The aspheric objective lens of the optical head with a numeric aperture (NA) of 0.6 has a focal length of $2.33 \mathrm{~mm}$. However, the NA 0.6 objective has a working distance of only $1.28 \mathrm{~mm}$, which may limit some measurement application. The working distance can be enlarged by replacing smaller NA objective lens. The NA 0.16 objective lens has a much longer working distance of $12 \mathrm{~mm}$. The linear detection range of the NA 0.6 and NA 0.16 objective lens is $6 \mu \mathrm{m}$ and $320 \mu \mathrm{m}$, respectively.

The full width at half-maximum (FWHM) $D_{w}$ of the focal spot is given by

$$
D_{w}=0.52 \frac{\lambda}{\mathrm{NA}} \text {. }
$$

The calculated $D_{w}$ of NA 0.6 and NA 0.16 objective lenses are $568 \mathrm{~nm}$ and $2.13 \mu \mathrm{m}$ (FWHM), respectively. The NA 0.6 objective lens with submicron laser spot is beneficial to detect deflections of submicrometer-sized cantilevers.

Additionally, the PDIC has a bandwidth of $80 \mathrm{MHz}$ $(-3 \mathrm{~dB})$ that is suitable to high-frequency detection. The $S_{\mathrm{FE}}$ calibration procedure can be done by giving cantilever a known vertical displacement directly. Overall, the ADS with NA 0.6 and NA 0.16 object lenses has a measurement sensitivity of $0.5 \mathrm{~nm} / \mathrm{mV}$ and $50 \mathrm{~nm} / \mathrm{mV}$, respectively. Because of higher measurement resolution, most of the measurements in this paper are carried out by the ADS with NA 0.6 objective lens.

The preamplifier is used to amplify and transform the signals from the PDIC into the focus error signal $S_{\mathrm{FE}}$ which is then processed by a high-speed 14-Bit DAQ card (PCI-9820, ADLINK) with a sampling rate up to $130 \mathrm{MHz}$ (ping-pong mode). Through the PCI bus, the digital data is analyzed by a program under a LabVIEW (National Instruments) platform. The acquired vibrational signal is processed by the fast Fourier transformation (FFT). Using the FFT algorithm, the acquired time domain signal is transformed into the frequency domain spectrum, which gives a clear overview of all resonant frequencies.

For improving the signal to noise ratio (SNR), microcantilevers are typically coated with high reflective materials like gold. However, the reflective layer may also cause binding of undesired molecules and might induce unwanted bimorph effects.

Furthermore, pure SU-8 surfaces can be employed for measuring thermodynamic properties of polymer thin films 


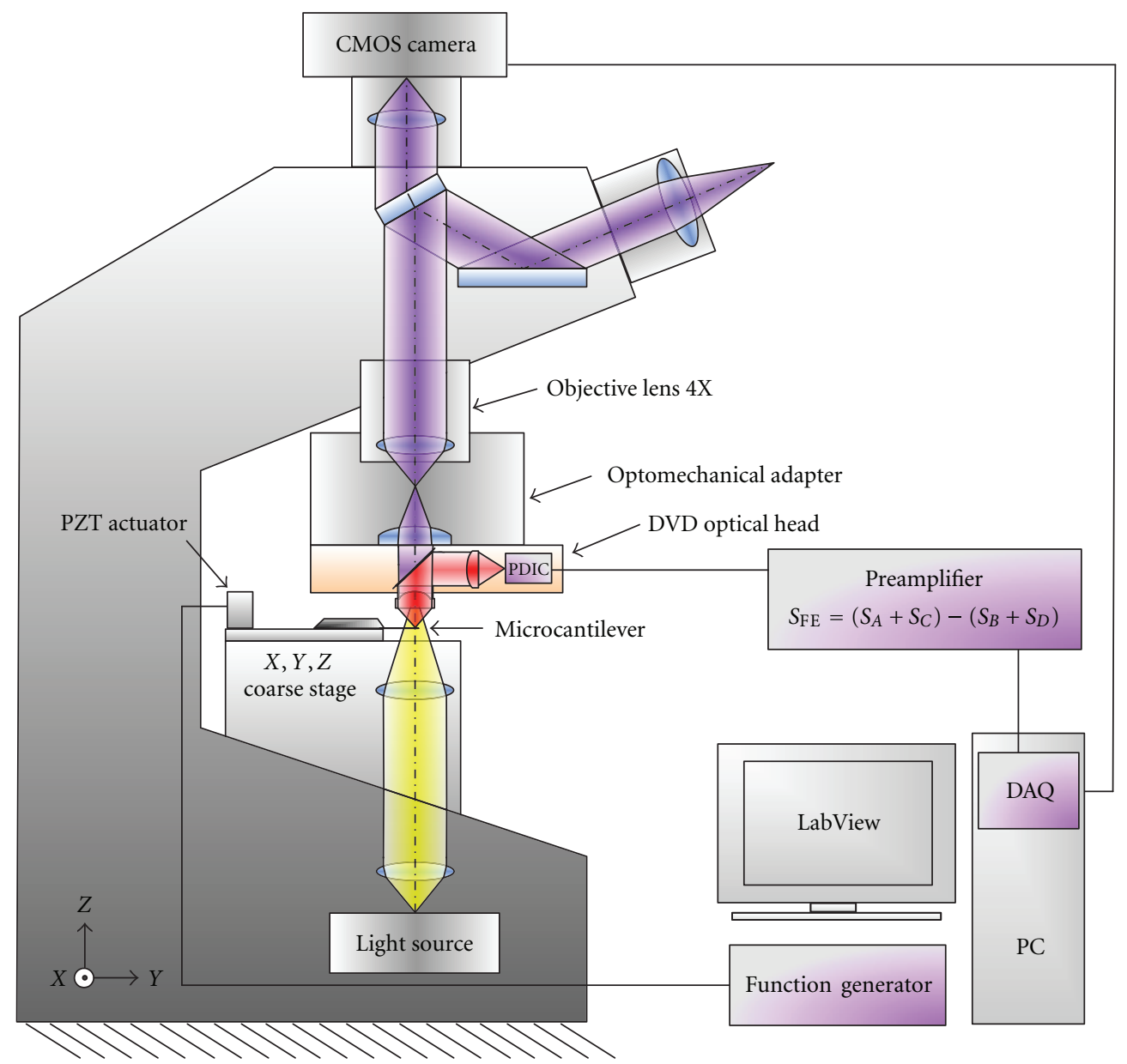

FIGURE 3: Block diagram of the ADS attached to an OM.

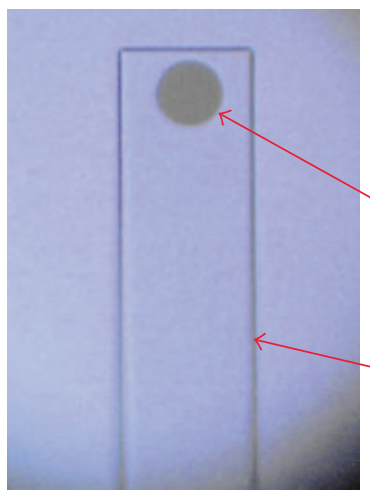

(a)

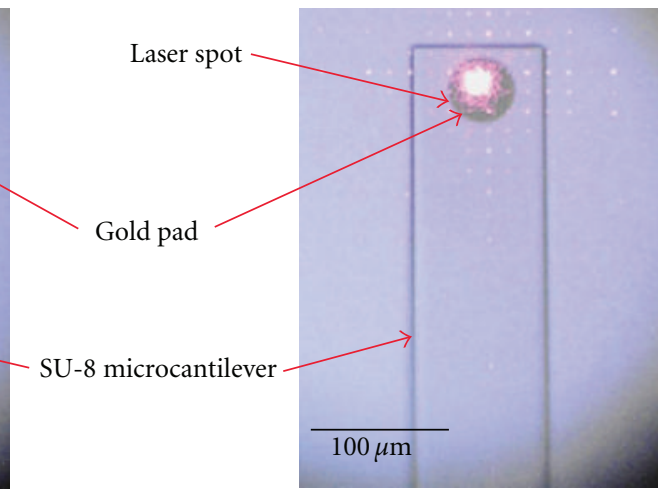

(b)

FIGURE 4: Microscopic images of (a) circular gold pad on SU-8 microcantilever and (b) focusing laser spot on gold pad.

deposed on the cantilevers surfaces [20]. Fast screening of the vibrational behavior of pure SU-8 microcantilevers would represent an extremely useful tool for the analysis of mechanical properties of polymeric material and for monitoring of degradation processes of biopolymers under various conditions [21].

The resonant frequencies of the microcantilevers are measured by the ADS in four conditions: with and without reflective coating; with and without external excitation. As expected, the SNR is strongly reduced when neither reflective pads nor external actuation is involved. The vibrational amplitude and the SNR of microcantilevers without reflective coatings can be significantly improved by the external excitation. In our experiments, a PZT actuator which is driven by a function generator is attached to a holder of the microcantilevers. 


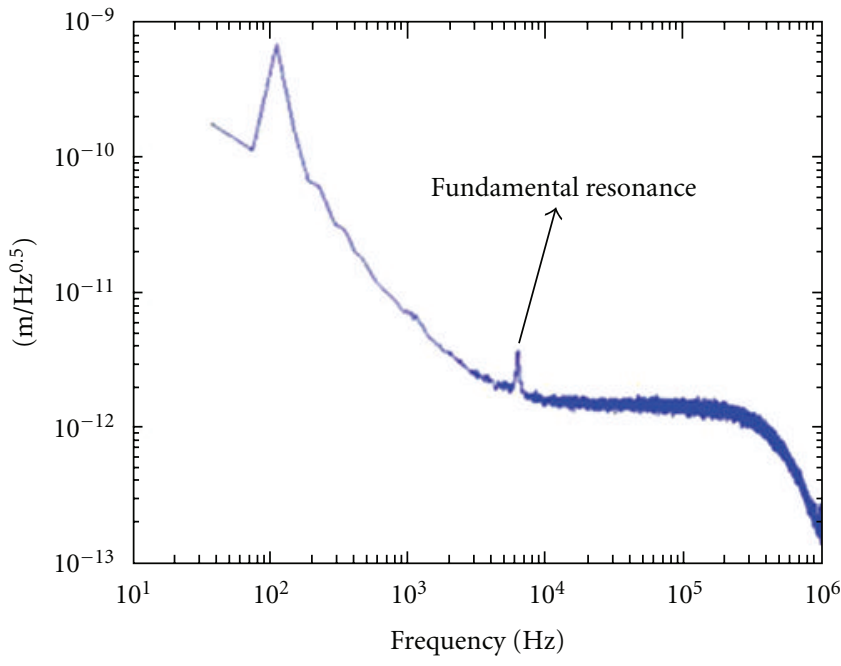

(a)

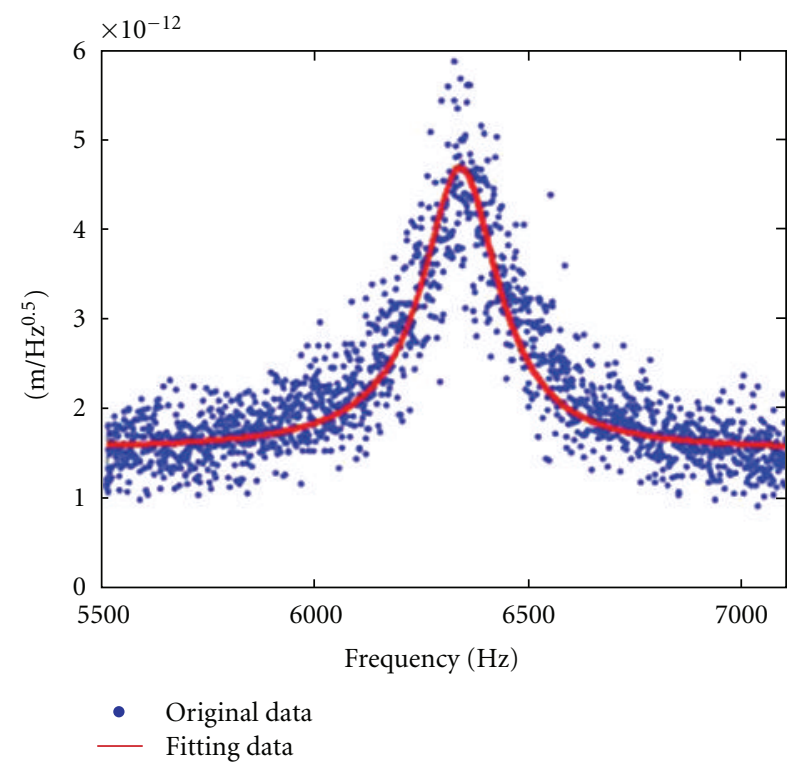

(b)

FIGURE 5: (a) Thermal noise spectrum and (b) SHO fitting curve for an SU-8 cantilever.

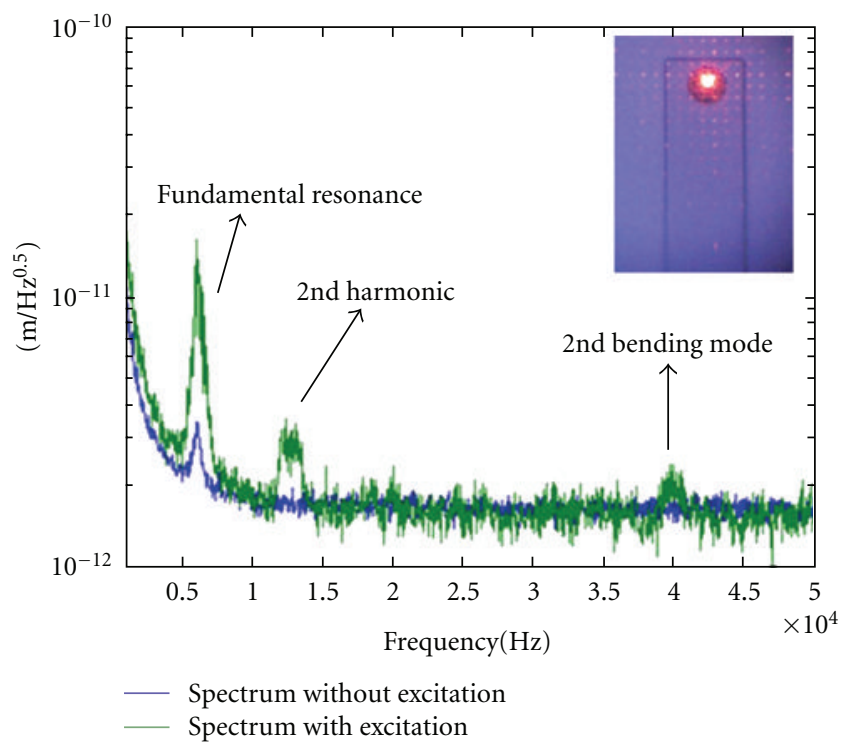

Figure 6: Thermal noise spectrum and excited spectrum by laser focused on gold pad.

Figure 4(a) shows an optical microscope image of the $20 \mathrm{~nm}$ thick circular gold pad on the free end of an SU-8 microcantilever. Through the $X, Y, Z$ coarse stages of the $\mathrm{OM}$, the laser beam is positioned and focused at the detected gold pad. And the focusing process can be simultaneously monitored as shown in Figure 4(b). The laser alignment can be adjusted with an $x-y$ resolution of $100 \mathrm{~nm}$.

\section{Results and Discussion}

Figure 5(a) shows the thermal noise spectrum and the fundamental resonant peak of the microcantilever. For measuring

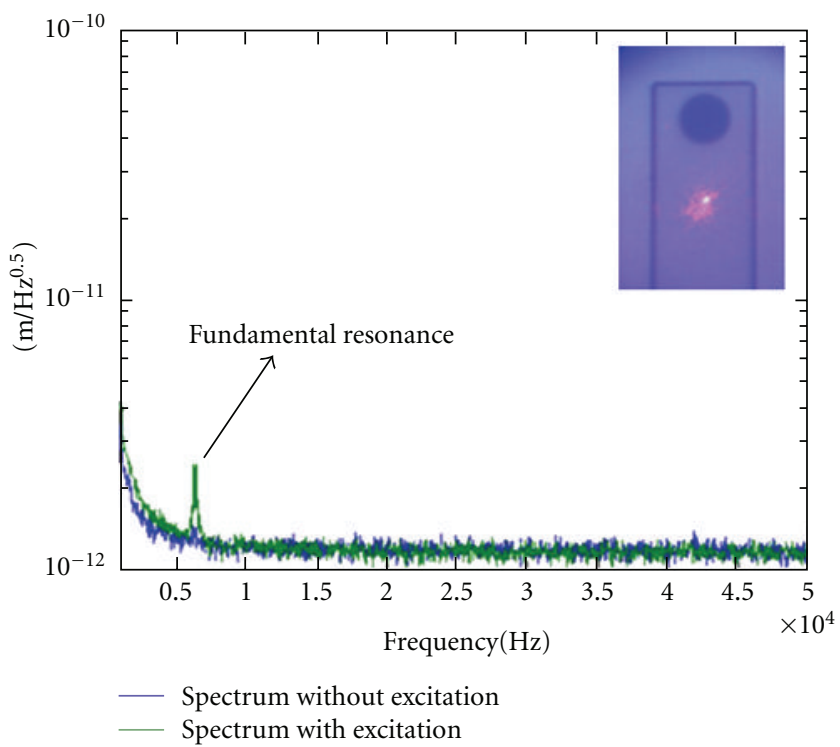

FIGURE 7: Thermal noise spectrum and excited spectrum by laser focused on the transparent part of the cantilever.

low resonance frequency cantilever, the bandwidth of the preamplifier we used is $1 \mathrm{MHz}$ only, thus the measured signal decayed in the end. For precise identifying the resonant peak, the spectral data are fitted by the simple harmonic oscillator (SHO) function as shown in Figure 5(b). The measured resonant frequency $6.328 \mathrm{kHz}$ is close to the calculated result range of $6.55 \pm 0.4 \mathrm{kHz}$ from (2) and the FEA simulated result of $6.59 \pm 0.5 \mathrm{kHz}$. The difference may be attributed to damping effects, which are neglected in the simplified theoretical models. Furthermore, the values for Young's modulus and density of the polymer depend on the SU-8 microfabrication-processing $[22,23]$ conditions 


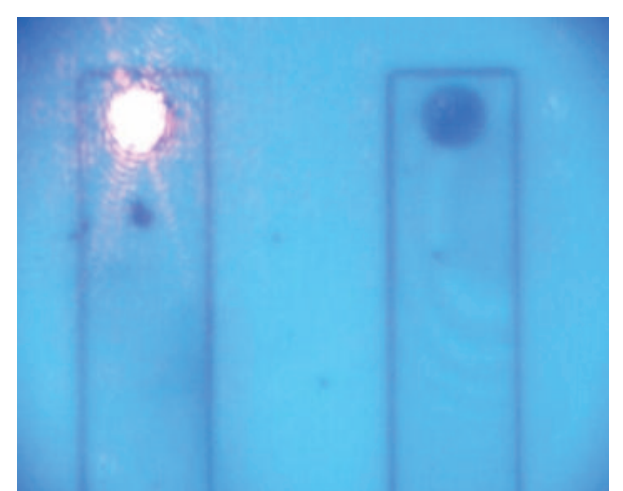

FIGURE 8: Microscopic images of focusing laser spot on the gold pad by using long working distance ADS.

might be slightly different than the ones presented in Table 1.

For enhancing free response vibration amplitude of the microcantilever, a mechanical excitation generated by the PZT actuator is applied. Figure 6 shows a comparison of the thermal noise spectrum and the excited spectrum when the laser is focused on the gold pad of the microcantilever. Using the external excitation, the first resonant peak becomes 4 times higher than without excitation. Also, the second resonant peak at $40 \mathrm{kHz}$ is intensified. The measured second resonant frequency is slightly smaller than the simulated value of $40.3 \mathrm{kHz}$. The $2 \mathrm{nd}$ harmonic is visible due to the external excitation and can be suppressed by decreasing the driving voltage of the PZT actuator.

Because of the low reflectivity of the SU-8 surface, the SNR of the $S_{\mathrm{FE}}$ is lower than that measured on the gold pad. Figure 7 shows the thermal noise spectrum and the spectrum with external excitation when the laser is focused next to the gold pad. The amplitude of the first resonant peaks is almost an order of magnitude lower than the one measured on the gold pad. The laser position is closer to the cantilever base compared to the previous measurements, but the decrease of vibration amplitude at this location is not significant enough to explain the decrease in signal strength. It is believed that the predominant contribution to the lower SNR of the $S_{\mathrm{FE}}$ and a lower $Q$ factor compared to the measurements on the gold pads is given by the low reflectivity of the SU-8 cantilever surface.

Nevertheless, the results demonstrate that the vibrational behavior of a homogenous microcantilever can be easily detected by the ADS even without the reflective coating and in presence of additional factors contributing to a reduction of the SNR. It is thus shown that the ADS has high capabilities in successfully characterizing the elastic properties of these polymer structures. Figure 8 shows an optical microscopic image captured by the ADS with NA 0.1 objective lens.

\section{Conclusions}

The ADS is capable of measuring the resonance frequency of SU-8 microcantilevers with and without reflective coatings.
The measured results coincide well with corresponding calculations and simulations. The ADS integrated with the OM and the CMOS camera makes the cantilever alignment and laser focusing efficient. For low reflective cantilever surface, the external excitation can be used to intensify the SNR.

Compared with the optical lever technique, the ADS has more advantages for high-speed microcantilever deflection measurements, such as compact size, easy adjustment, and high angular tolerance. Furthermore, the ADS with different objective lens is suitable for submicrometer cantilevers and long distance measurement applications. In future work, the ADS will be used to measure surface acoustic waves (SAWs) [24], which are widely applied in wireless devices as well as in chemical/biological sensors.

\section{Acknowledgments}

The authors would like to acknowledge support from the National Science Council of Taiwan (NSC95-3114-P-001008-MY3), Academia Sinica, and the Danish Council for Strategic Research (Xsense Project).

\section{References}

[1] J. Fritz, "Cantilever biosensors," Analyst, vol. 133, no. 7, pp. 855-863, 2008.

[2] S. Singamaneni, M. C. LeMieux, H. P. Lang et al., "Bimaterial microcantilevers as a hybrid sensing platform," Advanced Materials, vol. 20, no. 4, pp. 653-680, 2008.

[3] P. S. Waggoner and H. G. Craighead, "Micro- and nanomechanical sensors for environmental, chemical, and biological detection," Lab on a Chip, vol. 7, no. 10, pp. 1238-1255, 2007.

[4] J. Fritz, M. K. Baller, H. P. Lang et al., "Translating biomolecular recognition into nanomechanics," Science, vol. 288, no. 5464, pp. 316-318, 2000.

[5] J. W. Ndieyira, M. Watari, A. D. Barrera et al., "Nanomechanical detection of antibiotic-mucopeptide binding in a model for superbug drug resistance," Nature Nanotechnology, vol. 3, no. 11, pp. 691-696, 2008.

[6] N. V. Lavrik, M. J. Sepaniak, and P. G. Datskos, "Cantilever transducers as a platform for chemical and biological sensors," Review of Scientific Instruments, vol. 75, no. 7, pp. 2229-2253, 2004.

[7] S. Alexander, L. Hellemans, O. Marti et al., "An atomicresolution atomic-force microscope implemented using an optical lever," Journal of Applied Physics, vol. 65, no. 1, pp. 164$167,1989$.

[8] N. Blanc, J. Brugger, N. F. De Rooij, and U. Dürig, "Scanning force microscopy in the dynamic mode using microfabricated capacitive sensors," Journal of Vacuum Science and Technology B, vol. 14, no. 2, pp. 901-905, 1996.

[9] M. Tortonese, R. C. Barrett, and C. F. Quate, "Atomic resolution with an atomic force microscope using piezoresistive detection," Applied Physics Letters, vol. 62, no. 8, pp. 834-836, 1993.

[10] T. Itoh and T. Suga, "Development of a force sensor for atomic force microscopy using piezoelectric thin films," Nanotechnology, vol. 4, no. 4, article 007, pp. 218-224, 1993.

[11] P. A. Rasmussen, J. Thaysen, O. Hansen, S. C. Eriksen, and A. Boisen, "Optimised cantilever biosensor with piezoresistive read-out," Ultramicroscopy, vol. 97, no. 1-4, pp. 371-376, 2003. 
[12] R. Raiteri, M. Grattarola, H. J. Butt, and P. Skládal, "Micromechanical cantilever-based biosensors," Sensors and Actuators, $B$, vol. 79, no. 2-3, pp. 115-126, 2001.

[13] M. Alvarez, A. Calle, J. Tamayo, L. M. Lechuga, A. Abad, and A. Montoya, "Development of nanomechanical biosensors for detection of the pesticide DDT," Biosensors and Bioelectronics, vol. 18, no. 5-6, pp. 649-653, 2003.

[14] E.-T. Hwu, S.-K. Hung, C.-W. Yang, I.-S. Hwang, and K.-Y, Huang, "Simultaneous detection of translational and angular displacements of micromachined elements," Applied Physics Letters, vol. 91, no. 22, Article ID 221908, 2007.

[15] M. Nordström, S. Keller, M. Lillemose et al., "SU-8 cantilevers for bio/chemical sensing; fabrication, characterisation and development of novel read-out methods," Sensors, vol. 8, no. 3, pp. 1595-1612, 2008.

[16] F. G. Bosco, E. T. Hwu, C. H. Chen et al., "High throughput label-free platform for statistical bio-molecular sensing," Lab on a Chip, vol. 11, no. 14, pp. 2411-2416, 2011.

[17] E.-T. Hwu, S.-K. Hung, C.-W. Yang, K.-Y. Huang, and I.-S. Hwang, "Real-time detection of linear and angular displacements with a modified DVD optical head," Nanotechnology, vol. 19, no. 11, Article ID 115501, 2008.

[18] G. A. Matei, E. J. Thoreson, J. R. Pratt, D. B. Newell, and N. A. Burnahm, "Precision and accuracy of thermal calibration of atomic force microscopy cantilevers," Review of Scientific Instruments, vol. 77, Article ID 083703, 6 pages, 2006.

[19] S. S. Keller, L. Gammelgaard, M. P. Jensen, S. Schmid, Z. J. Davis, and A. Boisen, "Deposition of biopolymer films on micromechanical sensors," Microelectronic Engineering, vol. 88, no. 8, pp. 2297-2299, 2011.

[20] N. Jung, H. Seo, D. Lee, C. Y. Ryu, and S. Jeon, "Nanomechanical thermal analysis of the glass transition of polystyrene using silicon cantilevers," Macromolecules, vol. 41, no. 19, pp. 68736875, 2008.

[21] K. Yamashita, Y. Kikkawa, K. Kurokawa, and Y. Doi, “Enzymatic degradation of poly(L-lactide) film by proteinase $\mathrm{K}$ : quartz crystal microbalance and atomic force microscopy study," Biomacromolecules, vol. 6, no. 2, pp. 850-857, 2005.

[22] R. Feng and R. J. Farris, "The characterization of thermal and elastic constants for an epoxy photoresist SU8 coating," Journal of Materials Science, vol. 37, no. 22, pp. 4793-4799, 2002.

[23] M. Hopcroft, T. Kramer, G. Kim et al., "Micromechanical testing of SU-8 cantilevers," Fatigue and Fracture of Engineering Materials and Structures, vol. 28, no. 8, pp. 735-742, 2005.

[24] K. Länge, G. Blaess, A. Voigt, R. Götzen, and M. Rapp, "Integration of a surface acoustic wave biosensor in a microfluidic polymer chip," Biosensors and Bioelectronics, vol. 22, no. 2, pp. 227-232, 2006. 

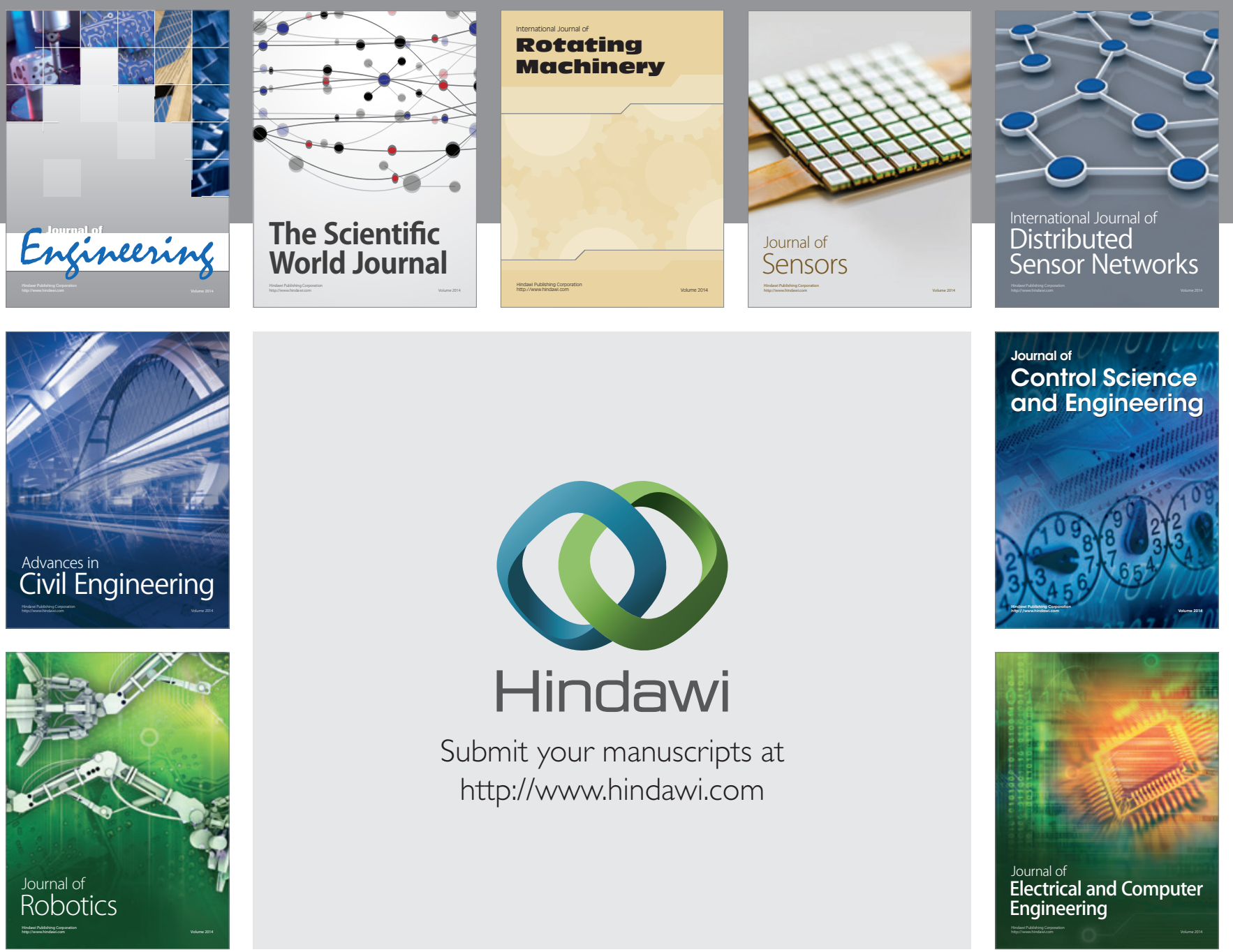

Submit your manuscripts at

http://www.hindawi.com
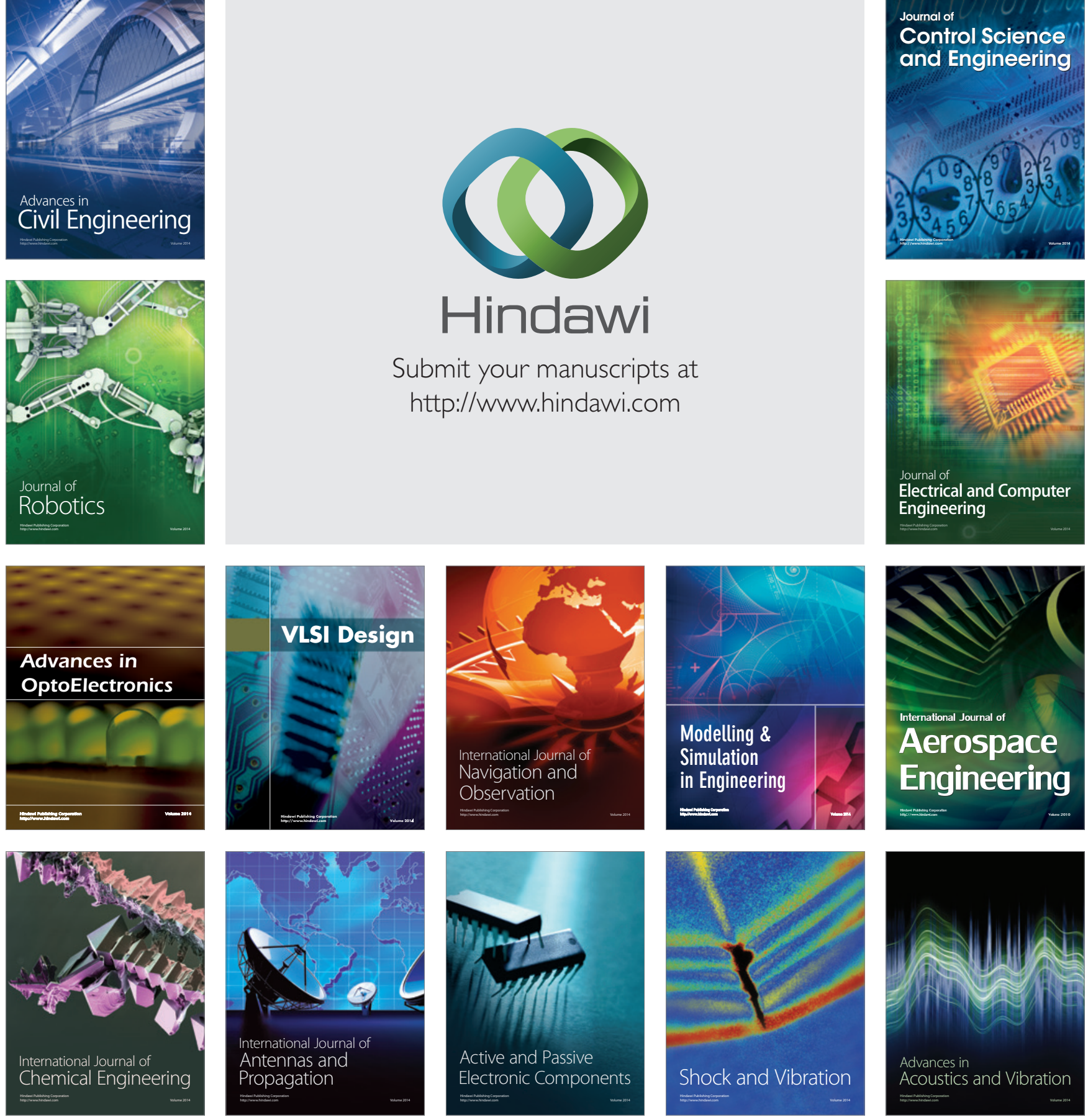